\title{
A Rare Cause of Right-Sided Air Bubble on Chest Radiograph: Intrathoracic Gastric Volvulus Related to Morgagni Hernia
}

\author{
Abdurrahman Sahin ${ }^{a}$ Hakan Artas $^{b} \quad$ Mehmet Yalniz \\ a Division of Gastroenterology \& Hepatology, Department of Internal Medicine, Firat \\ University Hospital, Elazig, Turkey; ${ }^{b}$ Department of Radiology, Firat University Hospital, \\ Elazig, Turkey
}

\section{Keywords}

Morgagni - Diaphragmatic hernia - Upside-down intrathoracic stomach · Intrathoracic gastric volvulus

\section{Abstract}

Morgagni hernia is a rare disorder in adulthood, and most of the cases are asymptomatic. Symptomatic cases are extremely rare and present with life-threatening complications. Early diagnosis and surgery are lifesaving. We hereby present an adult case of symptomatic Morgagni hernia. Diaphragmatic herniation of the stomach and mesenteroaxial rotation led to intrathoracic gastric volvulus in this case. A right-sided air bubble on a chest radiogram was the only finding leading to the suspicion of diaphragmatic hernia. Computed tomography in the diagnosis of diaphragmatic hernias is of great importance. 


\section{Case Reports in Gastroenterology}

Case Rep Gastroenterol 2017;11:103-108

(c) 2017 The Author(s). Published by S. Karger AG, Basel www.karger.com/crg

Sahin et al.: A Rare Cause of Right-Sided Air Bubble on Chest Radiograph: Intrathoracic Gastric Volvulus Related to Morgagni Hernia

\section{Introduction}

Diaphragmatic hernia is a herniation of the abdominal structures within the thoracic cavity due to a defect on the diaphragm and is primarily divided into congenital and acquired forms. Morgagni hernia occurs as a result of an anterior medial septum transversum defect due to failure of closure of the pars sternalis with the seventh costochondral arch. It is a rare condition accounting for $3-5 \%$ of the congenital diaphragmatic hernias. Most of the cases are diagnosed in newborns or in the early childhood. Morgagni hernia in adults is very rare and is often diagnosed incidentally. The relative rarity and vague presentation in most of the cases lead to delay in the diagnosis and treatment. Symptomatic adult cases may present with life-threatening complications such as acute obstruction, volvulus, or strangulation. Surgical repair of the diaphragmatic hernia is recommended in all cases to prevent the emergence of complications.

Radiologic assessment is helpful in the diagnosis and management of diaphragmatic hernias. Although plain radiography is the preferred technique, it may be normal in some cases [1]. Morgagni hernia may present as a homogeneous mass in the right cardiophrenic angle or as air bubbles in the thorax, originating from herniation of abdominal structures on a chest X-ray. Barium studies, fluoroscopy, computed tomography (CT), and magnetic resonance imaging are other helpful tools in the diagnosis of diaphragmatic hernias.

We herein report a case of Morgagni hernia diagnosed after the suspicion of herniation upon the findings of a chest radiograph who was diagnosed immediately and successfully rescued by surgical hernia repair.

\section{Case Presentation}

An 80-year-old female with a history of hypertension and cholecystectomy 10 years ago presented to the emergency department with complaints of epigastric pain, postprandial fullness, nonbilious vomiting of partially digested foods after eating, and self-induced vomiting. She had a weight loss for $12 \%$ of her body weight in the last month. She had been evaluated in another hospital outpatient clinic 10 days before presentation, and proton pump inhibitors had been prescribed. Her complaints had increased gradually over time and were more severe during the last week. She denied hematemesis, melena, hematochezia, fever, night sweats, or abdominal pain in the other parts of the abdomen.

On physical examination, the patient appeared anxious. Her skin turgor was decreased. Blood pressure was $120 / 75 \mathrm{~mm} \mathrm{Hg}$, pulse rate 86 beats/min, and body temperature $36.4^{\circ} \mathrm{C}$. Heart and chest examinations were normal. Abdominal examination revealed only epigastric tenderness. Blood tests revealed a mild renal dysfunction with urea $128 \mathrm{mg} / \mathrm{dL}$, creatinine $1.77 \mathrm{mg} / \mathrm{dL}$, and hypokalemia (2.8 $\mathrm{mEq} / \mathrm{L})$ (Table 1). A plain chest film showed an air bubble with air-fluid level in her right paracardiac region (Fig. 1). Enlargement of the common bile duct and main pancreatic duct was detected on abdominal ultrasound. The patient was discharged from the emergency department to the gastroenterology clinic for follow-up.

On the second day of hospitalization, the persistent epigastric pain increased, and the patient became intolerant to oral feeding. Total parenteral nutrition was started. An upper- 


\section{Case Reports in Gastroenterology}

Case Rep Gastroenterol 2017;11:103-108

(c) 2017 The Author(s). Published by S. Karger AG, Basel www.karger.com/crg

Sahin et al.: A Rare Cause of Right-Sided Air Bubble on Chest Radiograph: Intrathoracic Gastric Volvulus Related to Morgagni Hernia

gastrointestinal tract endoscopy revealed a twisted stomach, and the endoscope hardly passed through the distal part of the stomach (Fig. 2).

For excluding volvulus, hourglass stomach, or other pathologies related to this endoscopic finding, a thoracoabdominal CT scan was performed, and the CT images showed a Morgagni hernia, characterized by an anteromedial diaphragmatic defect with herniation of the transverse colon and the herniation of the distal part of the stomach into the thorax (Fig. 3). The patient was diagnosed as upside-down intrathoracic stomach related to Morgagni hernia and intrathoracic gastric volvulus. The patient then underwent laparoscopic mesh repair of the hernia defect and reduction of the stomach and other abdominal structures to the abdominal cavity. Her postoperative course was uneventful, and the patient was discharged on hospital day 12.

\section{Discussion}

Morgagni hernia was first described in 1769 and is an anatomical defect at the sternocostal junctions of the anterior diaphragm. Congenital fusion failure of the septum transversum leads to the development of Morgagni hernia. Morgagni hernia is the rarest form of congenital diaphragmatic hernias $(2-3 \%)$ and is mainly diagnosed in childhood in relation to respiratory symptoms [2]. Because the left hemidiaphragm is protected from herniation by the pericardial sac, the defect is on the right side in $80-90 \%$ of the cases [3].

Most of the cases are asymptomatic or show vague respiratory or gastrointestinal symptoms. Respiratory symptoms are more common in children. The herniation of abdominal contents is typically caused by an increase in the intra-abdominal pressure secondary to trauma, pregnancy, obesity, constipation, and chronic cough [4]. Symptomatic adult cases are extremely rare with less than 20 cases in the literature [5]. Life-threatening complications such as acute obstruction, volvulus, or strangulation are the presentations of acute cases [6]. Early diagnosis and urgent intervention are lifesaving in acute settings, as in this case.

Gastric volvulus is the twisting of all or part of the stomach by more than $180^{\circ}$ with obstruction of flow of material through the stomach. There are 3 categories of gastric volvulus: organoaxial volvulus, mesenteroaxial volvulus, and the combined form [7]. Organoaxial volvulus, which is the rotation of the stomach along the longitudinal axis, is the most common form and is associated with diaphragmatic disorders and paraesophageal hernia. Mesenteroaxial rotation is the rotation along the horizontal axis. Mesenteroaxial rotation and intrathoracic gastric volvulus related to paraesophageal hernia, which is called upside-down intrathoracic stomach, is a rare clinical entity [8].

In paraesophageal hernia, an air bubble is often detected in the mediastinum or left chest on a chest radiograph. As an unexpected finding, a right-sided air bubble on a chest radiograph should be reminiscent of Morgagni or Bochdalek hernias rather than hiatus hernias. On the other hand, CT is a reliable tool to diagnose Morgagni hernia and to distinguish it from other pathologies that manifest as the herniation of abdominal organs or structures into the thoracic cavity [4]. In the current case, CT examination was very helpful to differentiate Morgagni hernia from paraesophageal hiatal hernia. 
Sahin et al.: A Rare Cause of Right-Sided Air Bubble on Chest Radiograph: Intrathoracic Gastric Volvulus Related to Morgagni Hernia

The current report emphasizes that severe epigastric pain, the inability to tolerate oral feeding, and self-induced vomiting with the combination of abnormalities on a chest X-ray should alert clinicians for the presence of intrathoracic gastric volvulus in case of diaphragmatic hernia. In suspicion of any diaphragmatic hernia, CT should be performed to prevent delay in the diagnosis.

\section{Statement of Ethics}

The authors have no ethical conflicts to disclose.

\section{Disclosure Statement}

The authors have no conflicts of interest to declare.

\section{References}

1 Eren S, Ciris F: Diaphragmatic hernia: diagnostic approaches with review of the literature. Eur J Radiol 2005;54:448-459.

2 Minneci PC, Deans KJ, Kim P, Mathisen DJ: Foramen of Morgagni hernia: changes in diagnosis and treatment. Ann Thorac Surg 2004;77:1956-1959.

3 Schumacher L, Gilbert S: Congenital diaphragmatic hernia in the adult. Thorac Surg Clin 2009;19:469472.

4 Altinkaya N, Parlakgumus A, Koc Z, Ulusan S: Morgagni hernia: diagnosis with multidetector computed tomography and treatment. Hernia 2010;14:277-281.

-5 Razi K, Light D, Horgan L: Emergency repair of Morgagni hernia with partial gastric volvulus: our approach. J Surg Case Rep 2016;2016.

6 Loong TP, Kocher HM: Clinical presentation and operative repair of hernia of Morgagni. Postgrad Med J 2005;81:41-44.

7 Cribbs RK, Gow KW, Wulkan ML: Gastric volvulus in infants and children. Pediatrics 2008;122:e752e762.

8 Siow SL, Tee SC, Wong CM: Successful laparoscopic management of paraesophageal hiatal hernia with upside-down intrathoracic stomach: a case report. J Med Case Rep 2015;9:49. 


\section{Case Reports in Gastroenterology}

Case Rep Gastroenterol 2017;11:103-108

(C) 2017 The Author(s). Published by S. Karger AG, Basel

DOI: $10.1159 / 000457790$ www.karger.com/crg

Sahin et al.: A Rare Cause of Right-Sided Air Bubble on Chest Radiograph: Intrathoracic Gastric Volvulus Related to Morgagni Hernia

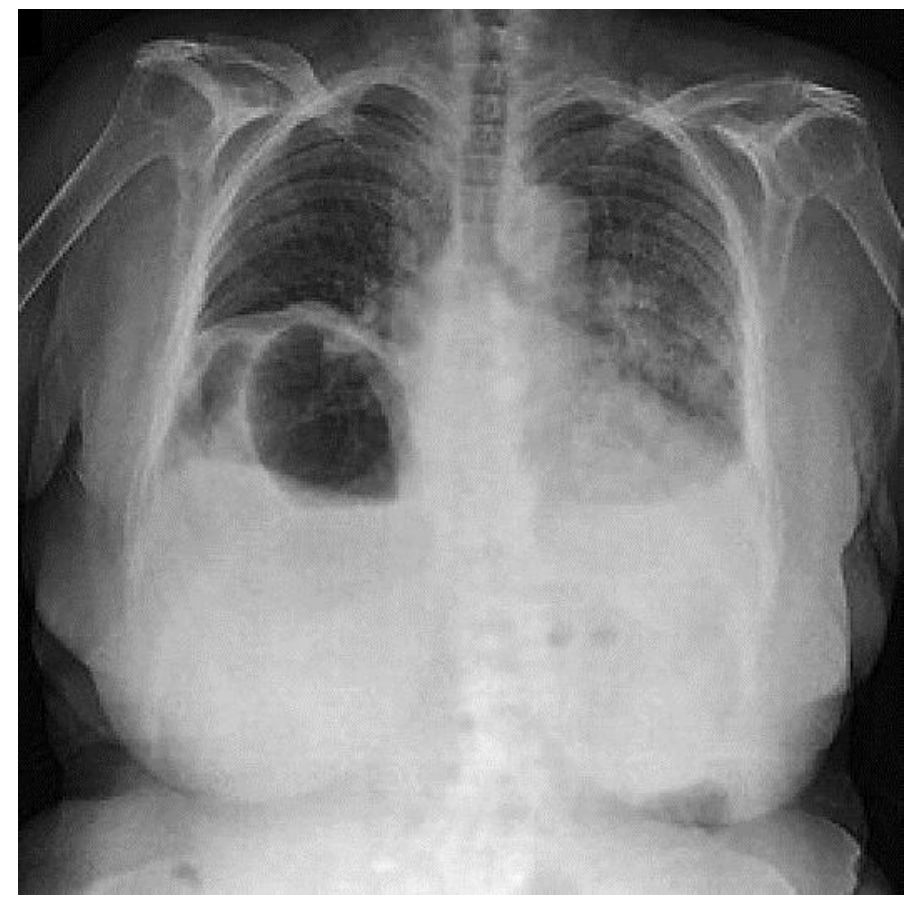

Fig. 1. Right-sided air bubble on a plain radiograph.

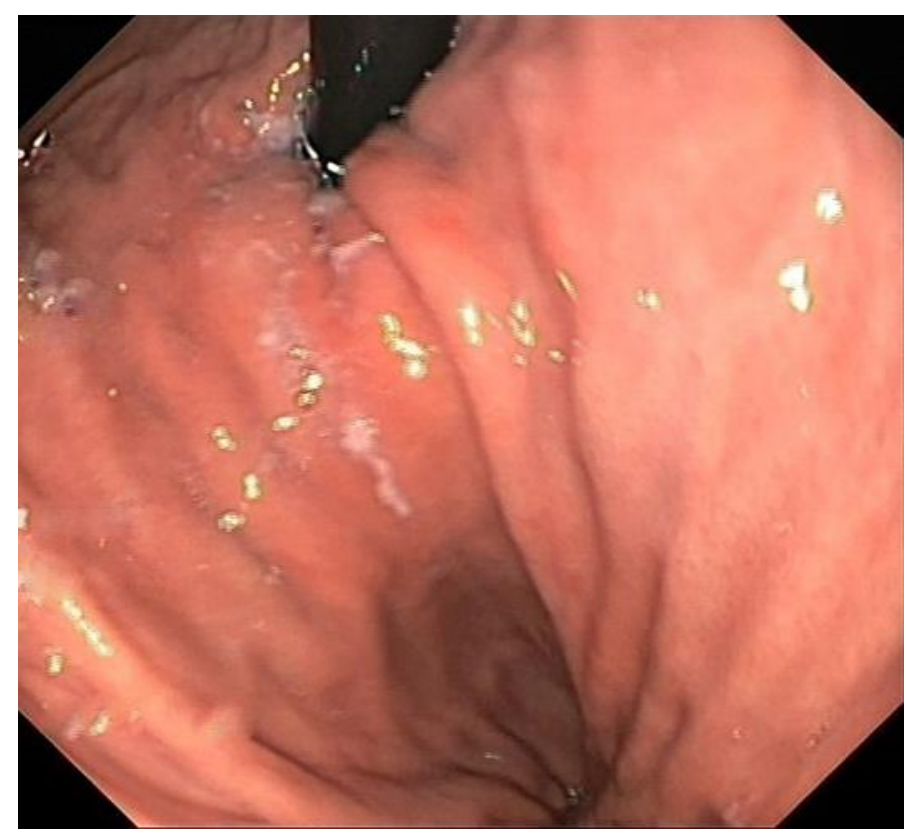

Fig. 2. Endoscopic view of a twisted stomach. 


\section{Case Reports in Gastroenterology}
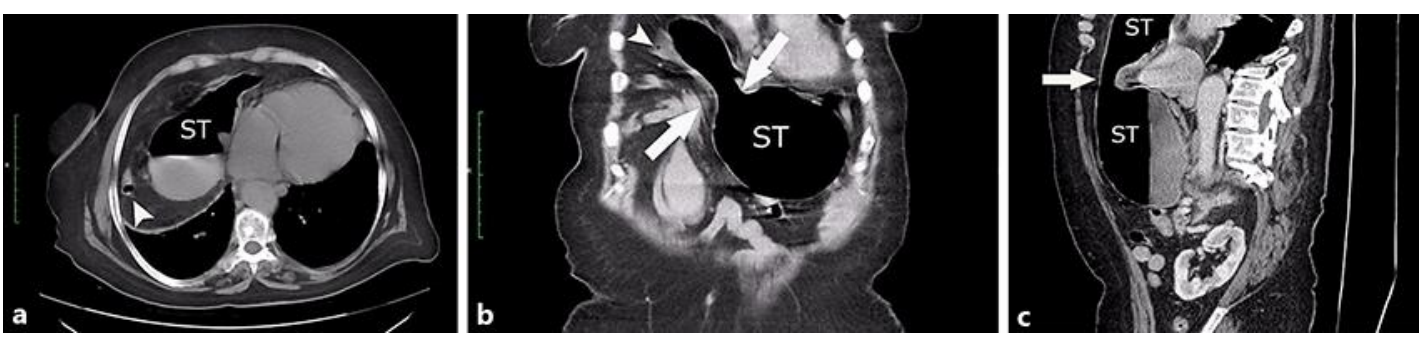

Fig. 3. Axial (a), coronal (b), and sagittal (c) images showing anteromedial diaphragmatic defect (white arrows) and intrathoracic herniation of the stomach (ST) and transverse colon (arrowheads).

Table 1. Laboratory values of the patient on admission

\begin{tabular}{lrc}
\hline Laboratory parameter & $\begin{array}{c}\text { Initial } \\
\text { value }\end{array}$ & $\begin{array}{l}\text { Reference } \\
\text { range }\end{array}$ \\
\hline White blood cells, $n / \mathrm{mm}^{3}$ & 7,600 & $3,800-8,600$ \\
Hemoglobin, g/dL & 13.7 & $11.1-17.1$ \\
Hematocrit, \% & 39.2 & $33-57$ \\
Platelet, $n / \mathrm{mm}^{3}$ & 359 & $140-360$ \\
Erythrocyte sedimentation rate, mm/h & 24 & $0-20$ \\
Fasting glucose, mg/dL & 114 & $75-115$ \\
Alanine aminotransferase, IU/L & 20 & $5-40$ \\
Aspartate aminotransferase, IU/L & 24 & $5-40$ \\
Alkaline phosphatase, IU/L & 62 & $30-120$ \\
Total bilirubin, mg/dL & 0.4 & $0.0-1.1$ \\
Amylase, U/L & 112 & $28-100$ \\
Lipase, U/L & 162 & $7-60$ \\
Urea, mg/dL & 128 & $10-50$ \\
Creatinine, mg/dL & 1.77 & $0.60-1.20$ \\
Sodium, mEq/L & 144 & $135-145$ \\
Potassium, mEq/L & 2.8 & $3.5-5.5$ \\
\hline
\end{tabular}

